\title{
Les élections municipales à Paris sous le Directoire
}

\section{Maurice Genty}

\section{(2) OpenEdition}

\section{Journals}

Édition électronique

URL : https://journals.openedition.org/ahrf/102

DOI : 10.4000/ahrf.102

ISSN : 1952-403X

Éditeur :

Armand Colin, Société des études robespierristes

Édition imprimée

Date de publication : 1 mars 2000

Pagination : 47-70

ISSN : 0003-4436

Référence électronique

Maurice Genty, «Les élections municipales à Paris sous le Directoire », Annales historiques de la

Révolution française [En ligne], 319 | janvier-mars 2000, mis en ligne le 11 mai 2006, consulté le 24 avril 2022. URL : http://journals.openedition.org/ahrf/102 ; DOI : https://doi.org/10.4000/ahrf.102

Ce document a été généré automatiquement le 24 avril 2022.

Tous droits réservés 


\title{
Les élections municipales à Paris sous le Directoire
}

\author{
Maurice Genty
}

1 Il n'est pas nécessaire de redire ce qui l'a été déjà tant de fois, que les historiens de la Révolution française ne se sont guère intéressés aux élections, et plus particulièrement sous le Directoire, à part J.-R. Suratteau pour l'élection des députés de l'an VI. Cela vaut surtout pour les élections autres que législatives, les élections municipales notamment, en particulier pour Paris. À vrai dire, sauf pour les élections de thermidor an IV, les sources, pour Paris, manquent le plus souvent ou n'existent que de façon très lacunaire ou indirecte. Pourtant, une étude des élections municipales à Paris sous le Directoire peut apporter quelques éléments susceptibles d'aider à la compréhension de la vie politique de cette période.

Le nouveau régime municipal et les nominations de l'an IV

2 La Constitution de l'an III, tout en organisant les municipalités de canton, avait prévu que la Commune de Paris, comme celle des autres villes de plus de cent mille habitants, en fait celles de Bordeaux, de Lyon et de Marseille, formeraient chacune un canton et seraient divisées en plusieurs administrations municipales, trois pour les trois dernières, douze pour la capitale, celles-ci correspondant aux douze arrondissements constitués après le 9 Thermidor par la réunion de quatre sections chacun; ces municipalités devaient être formées de sept membres élus par les assemblées primaires et renouvelés chaque année par moitié, à raison de quatre puis trois alternativement; l'unité de la commune devait être préservée par un bureau de trois membres nommés par l'administration départementale et confirmés par le Directoire exécutifíl. Le 19 vendémiaire an IV, une loi précisa que, "dans trois mois à compter du jour de la nomination du Directoire exécutif, ser(aie)nt nommés les membres du bureau cantonal" pour chacun de ces quatre cantons et que, "aussitôt que le bureau central sera(it) en fonctions, le département convoquera(it) des assemblées primaires de canton, pour l'élection de ces municipalités d'arrondissement" ${ }^{2}$, puis, le 4 brumaire, une loi rectificative ramena ce délai de trois à un mois ${ }^{3}$. Cependant, le 4 pluviôse, " une nouvelle loi prorogea ce délai au 1er thermidor an IV, par la considération que "les 
royalistes, vaincus le 13 vendémiaire dernier, nourriss(ai)ent encore le coupable espoir de renverser la liberté, que les factions terrassées par la courageuse Convention nationale, cherch(ai)ent à se relever de leur chute" et que, "à l'époque actuelle, la tenue des assemblées primaires de canton des communes de Bordeaux, Lyon, Marseille et de Paris, fourniroit un nouvel aliment aux discordes intestines"; en conséquence, elle chargeait le Directoire de nommer "provisoirement les membres qui (devaient) composer jusqu'à cette époque les administrations municipales de ces quatre communes. $»^{4}$

Le Directoire, apparemment, n'avait pas attendu cette loi pour préparer ces nominations, à en juger par un tableau donnant « la composition des municipalités de Paris, arrêtée par le Directoire exécutif le 6 nivôse de l'an IV »5. À vrai dire, sur quatrevingt-quatre officiers municipaux qu'aurait dû comprendre ce tableau, soixante-sept seulement étaient prévus. Cette préparation permit du moins au Directoire d'accélérer les choses lorsque fut votée la loi du 4 pluviôse, qu'il avait lui-même provoquée ${ }^{6}$.

Dès le 6 pluviôse ${ }^{7}$, il procéda aux nominations définitives, ou du moins présumées telles, sauf quatre; ce n'était que partiellement les mêmes que celles arrêtées le 6 nivôse ; trente noms seulement sur soixante-sept, soit $44,62 \%$, avaient été maintenus. La tâche du Directoire n'était pas pour autant terminée. D'abord, dix-neuf des citoyens pressentis refusèrent leur nomination ou démissionnèrent bientôt, tandis qu'un autre, ayant changé de domicile, n'avait pu être joint, et qu'un autre encore était décédé. Six furent remplacés dès le 22 pluviôse, mais, le 26, il manquait encore seize officiers municipaux, de sorte que six municipalités seulement étaient alors complètes ${ }^{8}$. Des citoyens continuèrent d'ailleurs à démissionner tandis que leurs remplaçants parfois refusaient ou démissionnaient à leur tour. Le Directoire eut ainsi à procéder à plusieurs reprises à de nouvelles nominations. Cinq destitutions furent en outre prononcées le 5 floréal, le Directoire s'apercevant que ses candidats n'avaient pas «les talens et les connoissances propres aux fonctions pour lesquelles il les avait appelés ${ }^{9}$, suivant une formule qui allait devenir courante. Le jeu des démissions et des destitutions devait se prolonger jusqu'en messidor, à la veille même des premières élections ${ }^{10}$.

Les élections de thermidor an IV ${ }^{11}$

5 Cinq mois après la mise en place des municipalités nommées par le Directoire, eurent donc lieu les premières élections municipales organisées dans le cadre de la Constitution de l'an III, les assemblées primaires ayant été convoquées pour le 1er thermidor conformément à la loi du 19 vendémiaire et à celle du 4 pluviôse an IV.

6 Peu d'innovations avaient été apportées aux modalités des élections elles-mêmes par rapport aux élections des débuts de la Révolution. La plus remarquable est celle qui avait multiplié le nombre des assemblées primaires; au lieu de quarante-huit, une par section, il y en avait à présent cent cinquante-huit, bien que le nombre des citoyens ayant le droit de vote se fût notablement réduit avec l'abandon du suffrage universel, mais alors qu'il s'était accru par rapport à la Constitution de 1791 avec l'octroi du droit de vote à tout contribuable; il s'agissait de limiter le nombre des participants éventuels à chacune d'elles, ce nombre ne devant pas dépasser celui de neuf cents, prévu par la Constitution, afin de faciliter le déroulement des opérations; le nombre de ces assemblées variait ainsi d'un arrondissement à l'autre, allant de neuf dans le IIe et le Xe à vingt-trois dans le XIIe.

7 Disposant de leur police intérieure, elles étaient en particulier « maîtresses de donner pour l'ouverture et la durée de leur scrutin le tems qu'elles jugeroient nécessaire ", 
comme le confirma le Département en réponse aux interrogations de plusieurs d'entre elles. Certaines en profitèrent pour retarder leur ouverture ou pour multiplier leurs séances. Ainsi, la 16e assemblée du VIe arrondissement ne se forma que dans l'aprèsmidi du 1er thermidor tandis que plusieurs autres, cinq sûrement, vingt-trois peut-être au total, attendirent le 2, d'autres le 3 , le 5 et le 6 , voire le 10 seulement pour la $8 \mathrm{e}$ assemblée du VIIIe arrondissement, faute d'avoir pu le faire le 1er, sans qu'il fût précisé pourquoi. Surtout, au lieu de recevoir les votes tout au long d'une seule journée, ce qui fut le cas pour six assemblées, la plupart décidèrent de le faire sur plusieurs jours de suite, en deux séances quotidiennes, le matin et en fin d'après-midi, deux jours pour quarante-cinq d'entre elles, soit $32,8 \%$, trois pour cinquante et une autres, soit $37,22 \%$, quatre pour vingt autres encore, cinq même pour sept assemblées, voire six pour la 7e assemblée du Ier arrondissement; il s'agissait ainsi de favoriser la participation des citoyens.

8 La procédure générale restait cependant la même qu'auparavant. L'assemblée était préparée par l'affichage des listes des ayants droit de vote et annoncée par affiches et au son du tambour. L'ouverture en était faite par un administrateur municipal ou par deux commissaires nommés par la municipalité concernée, puis lecture était donnée de la lettre de convocation du Département et des différents textes constitutionnels ou législatifs relatifs aux assemblées primaires; un bureau provisoire était constitué sous la présidence du doyen d'âge; les citoyens qui se présentaient devaient exciper de leurs titres, en soumettant à vérification leur carte de sûreté ou leur quittance d'imposition, voire les deux à la fois. On procédait alors à l'élection du bureau définitif, formé d'un président, de deux scrutateurs et d'un secrétaire, avant de passer à celle des officiers municipaux, par appel nominal des citoyens présents par ordre de numéro et, pour cette première fois, par scrutin de liste de sept noms.

9 Le zèle civique ne fut pas encore bien grand. Suivant un recensement effectué à partir des procès-verbaux des assemblées primaires, sur 119181 ayants droit de vote que comptaient les cent cinquante et une assemblées qui ont laissé des renseignements précis - il y en avait en principe 123259 pour l'ensemble de Paris ${ }^{12}$ - il n'y eut que 14503 votants effectifs; le taux de participation était donc seulement de $12,16 \%$. Ce nombre était comparable à ce qu'il avait été pour l'élection de Bailly à la mairie de Paris en août 1790 - 14010 -, mais avec un taux de participation plus élevé - il avait atteint $18,05 \%{ }^{13}$ -, supérieur à celui de l'élection de Pétion en novembre 1791 - 10632 -, mais avec un taux de participation du même ordre $-13,70 \%{ }^{14}$. Il semble cependant plus significatif de rapprocher l'élection de l'an IV de la première élection du conseil général de la Commune en septembre-octobre 1790; il n'y avait eu alors que 4638 votants pour le premier scrutin, 4518 pour le second ${ }^{15}$, soit $5,97 \%$ et $5,82 \%$ respectivement; la mobilisation apparaît donc à ce point de vue plus qu'honorable - elle avait plus que doublé -, révélatrice sans doute des espoirs suscités par le retour à des pratiques constitutionnelles normales, sans pour autant apparaître suffisante.

Les efforts en tout cas n'avaient pas manqué pour inciter les citoyens à venir voter. Outre la multiplication des assemblées primaires et la prolongation de leurs séances, la fixation de l'une des deux séances quotidiennes en fin d'après-midi dans nombre d'assemblées avait été expressément décidée à cette fin. Pour la 6e assemblée du Ve arrondissement, par exemple, il s'agissait «de faciliter aux ouvriers de venir après leurs travaux ", tandis que la 13e assemblée du Xe arrondissement déclarait que, fixer les assemblées au matin, c'était « priver nombre de fonctionnaires publics de participer 
aux nominations ». Dans le même espoir d'attirer les citoyens, la plus large publicité était donnée aux jours et heures des séances par l'affichage ou la proclamation au son du tambour, sinon par les deux moyens à la fois. Ces mesures n'eurent pourtant pas tout l'effet escompté et l'assistance resta limitée, quel que fût le moment de la journée. Ainsi, dans la 14e assemblée du XIIe arrondissement, s'il y eut trente-deux votants au total pour l'élection des officiers municipaux elle-même, ce fut au prix de plus de dix séances; s'il y en avait eu treize le 2 thermidor, entre trois heures et sept heures, pour la formation du bureau d'abord, un peu moins ensuite, dix seulement, pour l'élection des officiers municipaux, il ne s'en présenta qu'un le 3 thermidor, entre neuf heures $d u$ matin et deux heures de l'après-midi, mais douze le soir, de cinq à neuf heures, pas un seul le 4 de neuf heures à deux heures et cinq seulement de trois à neuf heures du soir, un seul encore le 5 entre neuf heures et deux heures et trois de cinq à neuf heures. D'un jour à l'autre, le nombre des citoyens qui répondaient aux incitations des assemblées allait généralement en diminuant; ainsi, dans la 14e assemblée du IVe arrondissement, il y eut 162 votants, presque un record, entre neuf heures du matin et sept heures du soir, mais quinze seulement les 3 et 4 thermidor et, dans la $8 \mathrm{e}$ assemblée du VIe arrondissement, leur nombre tomba de 56 le 2 thermidor à 17 le 3. Il est vrai que des citoyens, découragés par une attente trop prolongée, alors que le vote se faisait par appel nominal, se retiraient au cours du scrutin, sans avoir voté; ainsi, dans la $4 \mathrm{e}$ assemblée du Xe arrondissement, 66 citoyens s'étaient fait enregistrer le 1er thermidor, mais on ne releva que 51 bulletins, car « quinze autres, qui avaient été appelés plusieurs fois, s'étaient trouvés absents à la fermeture du scrutin »- phénomène déjà constaté en 1790 et 1791. D'un arrondissement à l'autre, d'une assemblée à l'autre, la participation fut naturellement fort diverse, sans être jamais très élevée; elle ne dépassa pas 17,66\% dans le IIe arrondissement, mais elle ne fut que de 7,77\% dans le XIIe; elle s'échelonnait d'un minimum de $2,63 \%$ dans la 18 e assemblée du XIIe arrondissement à un maximum de $31,2 \%$ dans la 1re assemblée du IIe. Elle avait également varié fortement entre l'élection du bureau définitif et celle des officiers municipaux; alors que, les bureaux paraissant susceptibles d'influencer le cours des opérations, voire l'orientation des votes, leur conquête pouvait sembler décisive, la participation était plus faible pour leur désignation que pour celle des officiers municipaux; avec un taux de $6,52 \%$, elle lui était de deux fois inférieure; les données, sans doute manquent pour un tiers des assemblées - cinquante-deux - mais c'est dans deux cas seulement qu'elle avait été plus forte que pour la formation du bureau ${ }^{16}$; dans trois cas seulement, elle était à peu près égale ${ }^{17}$; d'un minimum de $1,25 \%$ dans la 7 e assemblée du XIIe arrondissement, elle s'élevait à un maximum de $19 \%$ dans la 1re assemblée du Ier arrondissement; pour chaque arrondissement pris dans son ensemble, réduite à $3,26 \%$ dans le XIIe, elle ne montait qu'à 9,83\% dans le IVe; cela confirme le comportement qui avait été celui des citoyens parisiens dans les premières années de la Révolution, à la différence de ce que P. Gueniffey a relevé pour de nombreux départements ${ }^{18}$.

11 Non moins caractéristique, la dispersion des voix était aussi ample qu'en 1790-1791 en raison, cette fois encore, de l'absence de candidatures légalement reconnues. Sur centcinquante assemblées dont les procès-verbaux fournissent des données précises à cet égard, il était supérieur à celui des votants dans cent vingt-deux, soit 81,33\%; le maximum atteignait 63,84\% dans la 2e assemblée du VIe arrondissement, le minimum s'abaissant à $35,71 \%$ dans la $8 \mathrm{e}$ assemblée du IIe. Dans la 5e assemblée de ce dernier, pour 197 noms proposés, 108 n'avaient obtenu qu'une voix et 38 deux, mais 21 seulement dix et plus; dans la 7e assemblée du même arrondissement encore, sur 157 
noms sortis des urnes, 83 n'en avaient recueilli qu'une. Dans quatre-vingt-sept assemblées sur cent cinquante et une pour lesquelles les données sont connues, soit $57,61 \%$, la proportion des citoyens qui avaient obtenu une seule voix, qu'ils se soient ou non présentés à l'assemblée, dépassait $50 \%$ pour atteindre $74,45 \%$ dans la 2 e assemblée du XIIe arrondissement; elle n'était inférieure à $20 \%$ que dans un arrondissement, le Xe, où elle était de $17,82 \%$. Dans cent quarante assemblées, plus de $92 \%$, la proportion de ceux qui avaient recueilli deux voix dépassait $50 \%$ pour atteindre $88,7 \%$ dans la $7 \mathrm{e}$ assemblée du VIe arrondissement; elle était à peine inférieure au tiers dans deux assemblées seulement ${ }^{19}$. Ce n'était pas nécessairement pour eux-mêmes que les citoyens se prononçaient; ainsi, dans la 5e assemblée du VIe arrondissement, pour une liste de 90 présents, 58 , soit $64,4 \%$, n'avaient obtenu aucune voix, et 13 , soit $14,44 \%$, n'en avaient recueilli qu'une ou deux, mais 54 absents étaient dans le même cas. De même, dans la 12e assemblée du même arrondissement, pour une liste de 67 votants, 35, soit $52,23 \%$, n'avaient pas eu de voix, tandis que 35 absents en avaient obtenu au moins une. Parmi les absents qui recevaient des voix figuraient parfois des noms fantaisistes ou révélateurs de la position politique de ceux qui les avaient inscrits sur leurs bulletins, à moins qu'ils n'eussent voulu agir par dérision; c'est ainsi que, dans telle assemblée, on avait relevé le nom de Lafayette, celui de Cazalès dans telle autre et, dans une troisième, ceux du prince de Conti et de l'abbé Maury ${ }^{20}$. Dans dix assemblées seulement, soit $6,62 \%$, le pourcentage des citoyens qui avaient obtenu plus de voix était supérieur à $20 \%$, le maximum étant de $31,37 \%$, tandis que le minimum tombait à $2,04 \%^{21}$. Au total, pour ce premier tour de scrutin, treize officiers municipaux seulement, soit $15,47 \%$, à peine un sixième, pouvaient se flatter d'avoir obtenu la majorité absolue et de se trouver élus ${ }^{22}$; le mieux élu, dans le 8 e arrondissement, avait recueilli $65,20 \%$ des suffrages.

12 Un second tour de scrutin était donc nécessaire. Il devait être cette fois procédé dans chaque arrondissement à la formation d'une liste de " candidats ", c'est-à-dire la liste des citoyens qui avaient obtenu la majorité relative au premier tour, à raison de dix par poste à pourvoir, soit soixante-dix dans la plupart des $\operatorname{cas}^{23}$. Nous ignorons à quel moment précis les assemblées primaires furent de nouveau convoquées. Du moins connaissons-nous les résultats de ce second tour, grâce à un relevé officiel concernant l'ensemble des élus ${ }^{24}$. Comme dans les élections antérieures, la participation fléchit généralement; il n'y eut cette fois que 10905 votants, soit 8,8\% des ayants droit de vote; le maximum ne fut que de $15,31 \%$ dans le Ier arrondissement, où la participation avait exceptionnellement et légèrement augmenté, puisqu'elle n'avait atteint que $14,86 \%$ pour le premier tour, tandis que le minimum tombait à $6,04 \%$ dans le XIIe; la participation avait diminué de plus de moitié, de 1725 à 744 votants, dans le IVe arrondissement, de près de moitié, de 1029 à 555, dans le VIIIe, deux arrondissements, il est vrai, où il n'y avait plus que deux postes à pourvoir. Quinze officiers municipaux furent cette fois nommés à la majorité absolue, le mieux élu, dans le VIIIe arrondissement encore, ayant obtenu $74,05 \%$ des voix; les autres, au nombre de cinquante-six, durent se contenter de la majorité relative; le moins bien élu, dans le IXe arrondissement, n'avait recueilli que $16,12 \%$ des suffrages; cinq en avaient eu moins de $20 \%$.

13 Pour cette première expérience électorale dans le cadre du nouveau régime constitutionnel, les assemblées primaires s'étaient tenues sans problèmes notables. Lors du premier scrutin, outre la question concernant le droit des assemblées d'organiser librement leurs opérations, quelques difficultés avaient surgi quant aux 
limites de leurs circonscriptions, telle rue ayant été oubliée, telle portion de rue non indiquée expressément ${ }^{25}$. Si, par ailleurs, des affiches de convocation avaient été lacérées par la "malveillance $»^{26}$, il n'y avait eu que de rares incidents, au demeurant mineurs ${ }^{27}$. Pour l'ensemble des scrutins, rendant compte des élections au ministre de l'Intérieur, le 1er jour complémentaire de l'an IV, le commissaire du Directoire près le Département notait que, "en général, les assemblées primaires (avaient) été paisibles" et que "les suffrages avaient été donnés avec calme et liberté, à quelques exceptions près ${ }^{28}$. En particulier, «Dans le Ier arrondissement [signalait-il] deux partis s'étoient manifestés très ouvertement, l'un en faveur des Municipaux destitués, l'autre pour ceux nommés à leur place par le Directoire exécutif " - en fait, tous avaient été nommés, nous l'avons vu, à un moment ou à un autre. "Les votes du premier scrutin [précisait le commissaire du Directoire] étoient presque balancés entre ces deux partis; mais, au second, les Municipaux destitués (avaient) été exclus, et ceux qui les avoient remplacés (avaient) été élus à une majorité assez imposante "; effectivement, aucun des membres de la Municipalité nommés en pluviôse n'avait été élu, pas plus que quatre autres qui, le 21 ventôse, avaient remplacé ceux qui n'avaient pas accepté leur nomination; parmi les sept élus, quatre avaient été nommés le 4 prairial, un le 13 prairial et deux le 2 messidor seulement; l'un d'eux avait été élu au premier tour, avec $51,33 \%$ des suffrages, les autres au second tour, à une majorité en effet confortable, de $49,96 \%$ à $40,80 \%$, même si elle n'était pas absolue. "Dans le Ve arrondissement [poursuivait le commissaire du Directoire] il y a(vait) eu quelques mouvemens, mais sans suite » et «Dans le Xe arrondissement, le Commissaire de police de la Division de l'Unité (ancienne section des Quatre-Nations), a(vait) voulu troubler les assemblées primaires de sa Division », mais il avait été arrêté, ainsi que trois autres individus qu'il avait entraînés. En revanche, «Dans toutes les autres assemblées, le plus grand ordre a(vait) été maintenu». Le commissaire du Directoire ajoutait; «Le Directoire apprendra sans doute avec plaisir que la très grande partie des choix qu'il avoit fait (sic) ont été confirmés par le peuple». Un citoyen n'avait-il pas inscrit sur son bulletin ; "Éloge des Citoyens en place, et continuation de tous? " $^{29}$; était-ce une façon de dénoncer des manœuvres du pouvoir ou l'expression d'une constatation ou d'un vœu sincère? En fait, $50 \%$ des officiers municipaux avaient été reconduits dans leurs fonctions; si ce n'était pas le triomphe que le commissaire du Directoire annonçait à celui-ci, ce n'en était pas moins pour lui un succès. La situation, sans doute, variait d'un arrondissement à l'autre; dans quatre arrondissements seulement, outre le Ier où le succès était total, une majorité des officiers municipaux nommés par le Directoire avait été reconduite, six dans le VIIIe arrondissement, cinq dans le VIIe et quatre dans le IXe. Le contrôle par le Directoire de l'ensemble des municipalités parisiennes dépendait donc des tendances des nouveaux élus. Les choses lui parurent sans doute satisfaisantes; du moins n'avons-nous pas trouvé trace de destitutions prononcées contre des élus avant le coup d'État du I8 fructidor an V.

Des élections de germinal an $\mathrm{V}$ au coup d'État du 18 fructidor an $\mathrm{V}$

Dans l'intervalle, de nouvelles élections avaient eu lieu normalement en germinal an V. Il s'agissait alors de procéder au premier renouvellement partiel des municipalités parisiennes, quatre membres sur sept devant sortir par la voie du sort. De nouvelles modalités devaient entrer en application ${ }^{30}$ avec la possibilité de candidatures et l'introduction d'un double vote lors du second scrutin, l'un de nomination, l'autre de réduction, - en fait de censure; la liste des citoyens qui avaient obtenu la plus forte pluralité relative au premier tour ayant été établie, en nombre décuple des citoyens à 
élire, les membres des assemblées primaires devaient choisir simultanément ceux qu'ils désiraient élire et ceux qu'ils entendaient exclure. Nous manquons malheureusement de données sur ces élections, sauf pour quelques votes de cinq assemblées du IVe arrondissement ${ }^{31}$. Le premier scrutin eut lieu les 13 et 14 germinal, le second les 17 et 18; trois officiers municipaux furent élus au premier, le quatrième au second; il y avait eu 831, puis 704 votants, soit $7,73 \%$ et $6,55 \%$ des ayants droit, d'après un tableau dressé en messidor an IV $\mathrm{IV}^{32}$, contre $16,05 \%$ et $6,92 \%$ en thermidor an IV, soit une chute de près de moitié pour le premier scrutin, le taux de participation se maintenant à peu près pour le second ${ }^{33}$, mais peut-on généraliser cette tendance $?^{34}$ Quant à la dispersion des voix, nous n'avons de renseignements que pour deux assemblées; le pourcentage des noms sortis des urnes par rapport aux votants était, pour le premier scrutin, de 90,19\% dans l'une, de $67,24 \%$ dans l'autre, avec respectivement 46 noms pour 51 votants et 39 pour 58. Nous pouvons remarquer que, dans l'Almanach de l'an $\mathrm{V}$, figuraient tous les élus de thermidor an IV, à part trois exceptions ${ }^{35}$, mais quels furent ceux que le sort frappa ? Lesquels furent réélus, en dehors de trois des officiers municipaux élus dans le IVe arrondissement? Quels furent ceux qui remplacèrent les autres? Nous l'ignorons. Nous pouvons toutefois noter que, dans l'Almanach de l'an VI, publié avant que les conséquences du coup d'État du 18 fructidor aient pu être connues, nous retrouvons les mêmes noms que dans celui de l'an $\mathrm{V}$, sauf quinze nouveaux, soit $17,85 \%$, au nombre desquels ne figurait d'ailleurs pas celui du quatrième élu du IVe arrondissement.

Survint donc le coup d'État du 18 fructidor an V. Dès le lendemain, une loi déclara nulles, en particulier, toutes les opérations des assemblées primaires du département de la Seine, tous les individus nommés, " sans exception ", devant cesser aussitôt toutes fonctions ${ }^{36}$. Le Directoire, chargé par la même loi de nommer aux places ainsi devenues vacantes, procéda à cette opération, après consultation de ses commissaires près les différentes municipalités, sauf pour une place dans le Xe arrondissement, qui ne fut pourvue que le 16 vendémiaire an $\mathrm{VI}^{37}$. L'épuration fut massive; sur les quatre-vingtquatre administrateurs, seuls vingt-sept, un peu moins d'un tiers, $32,14 \%$, furent maintenus. Dans deux arrondissements, le Ve et le XIe, tous les administrateurs, sans exception, furent renouvelés, deux seulement dans quatre arrondissements ${ }^{38}$, trois dans trois autres ${ }^{39}$, quatre dans deux autres encore ${ }^{40}$ et cinq dans le VIe; quatorze d'entre eux, plus de la moitié, exactement $51,27 \%$, avaient été déjà nommés par le Directoire en l'an IV. Cependant, seize des nouveaux nommés, soit près d'un cinquième, $19,27 \%$, refusèrent leur nomination ou démissionnèrent bientôt ${ }^{41}$, de sorte que dix-neuf postes se retrouvèrent vacants, en y comprenant celui qui n'avait pas été pourvu le 23 fructidor.

D'ailleurs, dans une lettre écrite le 9 vendémiaire an VI par le commissaire du Directoire près le Département, les municipalités étaient « en général mal composées, ce travail ayant été demandé » le 18 fructidor «à 10 heures du soir pour le lendemain matin 9 heures »; le Bureau central du département et le commissaire du Directoire d'alors avaient cru « ne pouvoir mieux faire que de s'adresser aux Commissaires près les Administrations, mais (...) la plupart de ces Commissaires (étaient) ou royalistes ou sans caractère "; il semblait "donc important (...) de faire un rapport sur (ces) commissaires (...) et de présenter des candidats pour remplacer ceux des administrateurs qui n'(avaient) point accepté ou qu'il (était) essentiel de « destituer $»^{42}$. Le 13 vendémiaire, le ministre de l'Intérieur ayant approuvé cette suggestion ${ }^{43}$, le commissaire du Directoire proposa de nouveaux candidats ${ }^{44}$ et, le 21, le Directoire put compléter les administrations municipales, en confirmant ces propositions ${ }^{45}$; trois 
seulement de ces candidats avaient été déjà nommés en l'an IV, ce qui ramenait à 24 , soit $30,12 \%$ le nombre total des nouveaux administrateurs qui se trouvaient dans ce cas.

Les choses dès lors se stabilisèrent. Seules, cinq nouvelles nominations, semble-t-il, furent effectuées avant les élections suivantes, deux pour remplacer des citoyens qui avaient refusé leur nomination ${ }^{46}$, deux pour cause de démission ${ }^{47}$, le cinquième pour cause de destitution ${ }^{48}$.

Les élections de l'an VI

18 Les élections suivantes se déroulèrent comme prévu en l'an VI. Les modalités en avaient encore été modifiées; le principe des candidatures avait été supprimé le 24 pluviôse, celui du scrutin de réduction le 28 du même mois, puis, le 18 ventôse, avait été rétabli le système des trois scrutins de nomination, limités chacun à un jour, les deux premiers nécessitant la pluralité absolue ${ }^{49}$. Les citoyens durent cette fois nommer l'ensemble des administrations, " comme si la totalité des places (étaient) vacantes " ${ }^{50}$. Pas plus que pour les élections de l'an $\mathrm{V}$, nous ne disposons des procès-verbaux des assemblées primaires, sauf, toujours, celles du IVe arrondissement ${ }^{51}$ - encore n'en avons-nous les procès-verbaux intégraux que pour quelques-unes d'entre elles. Par eux, nous savons que les assemblées primaires, réunies pour la première fois le 1er germinal, conformément à la Constitution, n'eurent à procéder à l'élection des administrateurs municipaux qu'à partir du 7 ou 8 floréal, car elles avaient dû d'abord nommer leurs électeurs et les assesseurs des juges de paix et la loi du 25 fructidor avait prévu que, si « une assemblée primaire n'a(vait) pas terminé sa session le 19 germinal, elle s'ajournera(it) au 30 et ne tiendra(it) aucune séance durant la session de l'assemblée électorale", prévue du 20 au $29^{52}$. Dans le IVe arrondissement, trois officiers municipaux furent élus le 8 floréal, trois autres le 12, le septième le 14; d'un scrutin à l'autre, d'une assemblée à l'autre, la participation varia fortement; pour les assemblées dont le nombre des votants est connu, celui-ci allait de 27 à 104 pour le maximum, de 24 à 32 pour le minimum; pour l'ensemble de l'arrondissement, il allait de 523 lors du premier scrutin à 486 pour le second, puis à 707 pour le troisième, soit, en pourcentage, d'un minimum de $4,52 \%$ à un maximum de $6,58 \%$, si l'on se réfère au nombre des ayants droit de vote de l'an V, 10743 , mais de 7,63\% à $11,10 \%$ si l'on se réfère à celui de l'an VII, nettement moins élevé, 6364, chute difficile à expliquer; faut-il y voir l'effet du «serment de haine à la royauté et à l'anarchie » imposé aux citoyens par la loi du 19 fructidor an $V$ ? Cela rend en tout cas la comparaison avec les élections précédentes peu significative; cependant, le maximum, supérieur à celui de l'an $\mathrm{V}$ sur la même base - 6,58\% au lieu de 7,73\% -, était largement inférieur à celui de l'an IV, de deux fois et demie $-6,58 \%$ au lieu de $16,05 \%$. La tendance à la baisse s'était de toute façon poursuivie, mais qu'en avait-il été ailleurs? Nous n'avons qu'un autre renseignement pour le Ier arrondissement ${ }^{53}$, où il n'y aurait eu que 985 votants, soit $11,5, \%$ sur la base des ayants droit de l'an V, 10,37\% sur la base des ayants droit de l'an VII, alors qu'il y en avait eu 1311 en thermidor an IV, soit 15,31\% des ayants droit, ce qui représente un recul d'un quart, 24,87\%. Quant à la comparaison entre la participation pour la nomination du bureau et celle pour l'élection des administrateurs, nous n'avons de renseignements que pour deux assemblées du IVe arrondissement, échantillonnage beaucoup trop faible pour être significatif; notons toutefois que le nombre des votants y fut cette fois plus élevé pour la désignation du bureau que pour celle des administrateurs, de deux à quatre fois plus ${ }^{54}$. 
19 Avec la suppression des candidatures, la dispersion des voix reparut avec force, mais sans atteindre l'ampleur de l'an IV. Pour un total de 948 votants connus pour l'ensemble des scrutins, 805 noms sortirent des urnes, soit $84,9 \%$, sur lesquels 371 , soit $46,08 \%$, n'obtinrent qu'une voix, alors que 142 seulement, un peu plus du sixième, $17,63 \%$, en réunissaient dix et plus. D'un scrutin à l'autre, la proportion variait; pour les quatre assemblées qui permettent une comparaison suivie ${ }^{55}$, tandis que le nombre des votants reculait de 259 à 156 pour remonter à 284 , alors pourtant qu'il n'y avait plus qu'un administrateur à nommer, le nombre des noms proposés passa de 182 , soit $70,27 \%$, à 113 , soit $72,4 \%$, pour retomber à 58 , soit $20,42 \%$; le nombre des citoyens qui n'avaient obtenu qu'une voix recula, de son côté, de 100 , soit $54,94 \%$ des noms sortis des urnes, à 45 , soit $40,7 \%$, puis à 29 , soit $10,21 \%$.

Les élections n'en furent pas moins âprement disputées. Nous savons en effet que les démocrates, en particulier, firent un gros effort de mobilisation et de propagande en constituant des cercles constitutionnels. Plusieurs pièces, qui ne sont peut-être pas à prendre au pied de la lettre, dénoncèrent par ailleurs l'atmosphère qui régna dans diverses assemblées, notamment un rapport établi par le commissaire du Directoire près le canton de Paris, sous la forme d'un tableau accompagné d'un brouillon de "résumé" de ce tableau ${ }^{56}$, pièces concernant, il est vrai, la nomination des électeurs, effectuée en premier. Il en résulte que, sur les douze administrations municipales, neuf avaient été " sous le joug du terrorisme et de l'anarchie; - Que dans trois seulement les choix (avaient) été mélangés, mais que, dans les neuf autres (avaient) figuré exclusivement les ex-membres des Comités révolutionnaires, des Jurés révolutionnaires, des Septembriseurs, des partisans de Babeuf, des acquittés de Vendôme ${ }^{57}$ et que, par la force, la violence, les Voyes de fait, leur Coalition impie et l'introduction dans leurs assemblées d'hommes qui ne payant pas de Contribution, n'avaient pas le droit de voter, ils s'(étaient) institués les directeurs suprêmes des Élections ». À l'en croire, les assemblées primaires s'étaient données des bureaux " composés des deux extrêmes »; tel président d'une assemblée ${ }^{58}$ avait été "l'un des principaux acteurs de la conspiration de vendémiaire, tel autre avait été "envoyé à la haute cour de Vendôme ${ }^{59}$. Les élections n'auraient été faites régulièrement que dans trois arrondissements, les VIIe, IXe et XIIe. Des troubles s'étaient produits dans onze assemblées, ce qui était finalement peu - 6,96\% -, avec violences dans quatre d'entre elles $^{60}$; dans la $2 \mathrm{e}$ assemblée du Ier arrondissement, deux citoyens avaient été « frappés, chassés du lieu des séances" et s'étaient retirés, "les habits déchirés et le corps portant les marques des coups et contusions qu'ils (avaient) reçus». Ces troubles avaient entrainé des scissions dans cinq assemblées ${ }^{61}$, une sixième ayant réussi à y mettre fin $^{62}$. À défaut de troubles, des manœuvres avaient été perpétrées soit par des royalistes, soit par des " anarchistes ", ou encore par les uns et les autres à la fois; ainsi, dans le Ier arrondissement toujours, si les royalistes s'étaient "peu montrés », les anarchistes étaient « apparus avec force », se livrant à une "fabrique de scrutins qui se distribuoient dans le cabaret le plus proche de chaque assemblée; et distribution faite avec une telle profusion que le lendemain de la dernière séance, les scieurs de bois des chantiers, rue Miromesnil en (avaient) fait beaucoup voir qu'ils avoient de reste ». Dans le IIIe arrondissement, les deux partis s'étaient en revanche affrontés, il est vrai «par ruse, non par violence ", mais la nature de cette "ruse " n'était pas précisée. Dans la 11 e assemblée du IVe arrondissement, " on obligeoit les citoyens de recommencer sans cesse les opérations pour les fatiguer et les dégoûter »- accusation plus d'une fois portée contre les assemblées dans la première période de la Révolution. Dans deux 
assemblées du VIIIe arrondissement ${ }^{63}$, on avait introduit des «individus n'ayant pas d'inscription civique, et qui néanmoins (avaient) été admis à voter sous la seule exhibition de leur carte de sûreté ». Dans la 5e assemblée du XIe arrondissement, enfin, des bulletins avaient été préparés, des "vins distribués" par des individus qui avaient expulsé de l'assemblée les autres citoyens, lesquels s'étaient réunis ailleurs. Une autre pièce, un "tableau des membres composant les administrations Municipales du Département de la Seine en messidor an VI ${ }^{64}$, confirmait et précisait ces indications. Si les élections ne s'étaient faites régulièrement que dans les trois arrondissements déjà indiqués, elles paraissaient cependant valides dans trois autres, les IIIe, IVe et Ve, qui n'avaient pas connu de scissions. Il n'en était pas de même ailleurs. Le Ier arrondissement, qui avait été déjà fort disputé en thermidor an IV, s'était particulièrement distingué; «Il est de notoriété [était-il écrit] que les sept administrateurs ont été portés sur une liste arrêtée au Cercle constitutionnel (de la Conception, rue Honoré)» - c'est cette liste qui aurait été distribuée auprès des ouvriers de la rue de Miromesnil; « aussitôt l'ouverture des assemblées, si les émissaires de ce cercle étoient arrivés les premiers, on procédoit sur-le-champ au scrutin; si c'étoit le contraire, ils obtenoient à force de cris et de menaces que le scrutin seroit ouvert jusqu'à 6 heures du soir, et ils alloient faire recrue [...]; les Républicains paisibles (avaient) été écartés par la présence des anciens membres des Comités révolutionnaires et autres gens » de cette espèce, de sorte que «masse de citoyens épouvantés de cette apparition a(vait) quitté sur-le-champ les assemblées primaires », et pourtant, précisait une autre pièce ${ }^{65}$, alors que « les 12 assemblées avoient été presque désertes ", il s'était " trouvé 945 votants "; il est vrai, à en croire les administrateurs du Département ${ }^{66}$, que les «émissaires » du Cercle constitutionnel n'avaient "pas eu une influence égale » partout; dans les divisions du Roule et des Champs-Élysées, ils n'avaient pas eu « un succès complet » et c'était « dans les deux autres divisions (celles des Tuileries et de la Place-Vendôme) qu'avaient été choisis les sept administrateurs actuels ». Dans le VIe arrondissement, les assemblées primaires avaient été, elles aussi, "fortement tourmentées ", mais à la fois par les "anarchistes » et par les royalistes; il paraissait "aussi qu'on a(vait) admis à voter des citoyens qui n'en avaient pas le droit, ou du moins qui n'étaient pas inscrits sur le registre civique ", mais, était-il ajouté, l'instruction officielle adressée aux assemblées primaires de Paris «étoit un peu amphibologique sur ce point, et toutes les Élections de Paris seroient dans le cas d'être annulées, si on les jugeoit à cet égard avec beaucoup de sévérité ». Dans le VIIIe arrondissement, un seul incident, minime, était à signaler, de sorte qu'on ne croyait " pas que ce (pût) être un moyen de nullité » ${ }^{67}$. Dans le Xe arrondissement, en revanche, il y avait eu une foule d'irrégularités, de violences et d'infractions à la loi, concourant à rendre nulles toutes ces Élections ». Dans le XIe arrondissement, enfin, une scission s'était produite dans deux assemblées ${ }^{68}$, et "l'intrigue (était) entrée pour beaucoup dans la nomination des administrateurs municipaux; pour s'en convaincre, il suffis(ai)t de rappeler que dans plusieurs assemblées (...), on a(vait) affiché sur la boëte au scrutin la liste des administrateurs actuels, avec le Titre liste des administrateurs nommés ou à renommer."

21 Quels furent en tout cas les résultats? Nous connaissons le nom des élus par un tableau établi en messidor en vue d'une épuration ${ }^{69}$. Sur les quatre-vingt-quatre nouveaux administrateurs municipaux, trente-quatre avaient été déjà nommés ou continués par le Directoire en fructidor an $\mathrm{V}$, soit $40,47 \%$, dont quatre dans les VIe et XIe arrondissements, cinq le Ve, pas moins de six dans le IXe et sept dans le XIIe, mais 
aucun dans le Ier et dans le IVe ${ }^{70}$. Si cette fois encore, ce n'était pas un triomphe pour le Directoire, c'était tout de même un large succès, d'autant plus qu'il pouvait compter sans doute sur un nombre plus ou moins important de nouveaux élus. Certains de ces administrateurs, il est vrai, ne s'étaient pas toujours révélés sûrs ou allaient cesser de donner satisfaction - ce fut le cas pour quatre administrateurs du Xe arrondissement ${ }^{71}$. Cinq d'ailleurs avaient été déjà destitués en fructidor ${ }^{72}$. Si, par ailleurs, vingt-quatre des nouveaux administrateurs, soit $28,57 \%$, avaient déjà figuré dans l'Almanach de l'an VI, soixante-et-un, près des trois-quarts, devaient encore figurer dans celui de l'an VII, mais l'Almanach ne pouvait tenir compte des changements survenus après sa publication. Quelques-uns, au nombre de neuf, dont quatre dans le seul VIIe arrondissement, certains, il est vrai, sous menace de destitution, devaient en effet démissionner ${ }^{73}$.

Une épuration avait d'ailleurs été envisagée très tôt. Dès le 19 prairial, le ministre de l'Intérieur chargeait le commissaire du Directoire près le Département de la préparer ${ }^{74}$ et, le 24 messidor, celui-ci adressa au Directoire le tableau des administrateurs municipaux cité plus haut, accompagné de renseignements sur chacun d'eux et de propositions sur le parti à prendre à leur égard, ainsi que du nom des candidats destinés à remplacer ceux qu'il conseillait de destituer ${ }^{75}$. L'épuration prévue resta cependant limitée; elle ne toucha que quinze administrateurs, soit $17,88 \%$. Dans six arrondissements, un sur deux ${ }^{76}$, tous les élus étaient à conserver; pour le IIe, où, en raison de la scission survenue dans la première assemblée primaire, la municipalité n'avait pas encore été installée, "en attendant la décision du Corps Législatif ", rien n'était proposé; une seule destitution était conseillée dans le IIIe arrondissement, deux dans le Ve, mais pas moins de quatre dans le Ier, le XIIe et le XIe, où les assemblées avaient été les plus agitées. Les administrateurs du Ier arrondissement étaient particulièrement visés, eux dont l'élection aurait été préparée au cercle constitutionnel de la Conception. Le premier élu, Perdry, « tour à tour royaliste et terroriste, dénué de moyens, mais intrigant très adroit », pour qui "rien n'(était) sacré quand il s'agissait d'arriver à ses fins ", n'avait d'autre plan que « d'aller au département pour s'occuper de ses affaires et corriger les vices de la fortune ». Le second, Foloppe, un apothicaire demeurant rue Honoré, « ami de la parfaite égalité, du nivellement des fortunes », était, «en dernière analise (sic), également partagé du côté de la folie et de la sottise », sa folie étant prouvée par le fait «qu'il applaudissait à toutes les charrettes des victimes qui passoient devant sa porte, quoique son père fût du nombre " - dans toutes les charrettes?; les administrateurs du département ajoutaient qu'il fréquentait "habituellement Antonelle et autres individus». Le troisième, Bunon, qui avait " arrosé les exclusifs de plus de trois cens bouteilles de vin », avait été « le fidèl (sic) ami d'Henriot ", avec lequel il avait fait « ses premières armes aux Barrières " (la veille du 14 juillet 1789); il avait poussé «dans le tems le zèle si loin que, dans la section des Champs-Élysées, il aurait désiré une guillotine "à la porte de l'assemblée, offrant ses services au cas où il manqueroit un bourreau ». Le quatrième, Marquet, était un admirateur d'Hébert, le cinquième, Brullart, un "terroriste éhonté, dénonciateur à gages ", tandis que le sixième, Moussard, se vantait d'être l'émule de Robespierre et prétendait imposer "aux enfants l'obligation de dénoncer leur père " - il était instituteur - « jurant que s'il apprenait que son père fût aristocrate, il le poignarderoit lui-même "; quant au septième, Bazelot, " ami particulier d'Antonelle ", son nom seul inspirait « la terreur, par le souvenir des scènes sanglantes où on l'a(vait) vu figurer » les massacres de septembre sans doute. Pourtant, quatre seulement étaient proposés 
pour la destitution, Perdry, Bunon, Foloppe et Bazelot. Que valaient en effet les accusations portées contre les sept élus ${ }^{77}$ ? À plusieurs reprises, les quatre derniers élevèrent des réclamations et Perdry produisit « les témoignages les plus favorables » de plusieurs représentants du peuple, parmi lesquels $\mathrm{Cabanis}^{78}$, mais en vain; ils furent destitués le 27 messidor, remplacés par les candidats proposés par le commissaire du Directoire et qui avaient été déjà nommés en nivôse an IV. Le Directoire ne réagit pas aussi rapidement aux autres propositions qui lui étaient faites; il attendit le 5 ventôse an VII pour décider la destitution de quatre élus du Xe arrondissement, qui avaient été déjà suspendus par l'administration du département ${ }^{79}$, de même que celle de quatre administrateurs du XIe arrondissement, qui avaient "grossièrement intrigué pour se faire continuer en fonctions ${ }^{80}$. D'autres changements eurent lieu, sans que l'on puisse toujours les dater précisément. Au total, ce sont trente et un élus de l'an VI qui furent remplacés, soit 36,9\%, parmi lesquels douze seulement par destitution, du moins effectivement connue, sans compter une treizième destitution provisoire, soit $15,47 \%$, moins du sixième; encore ces changements s'étaient-ils échelonnés sur de longs mois, de thermidor an VI à ventôse an VII, à la veille de nouvelles élections.

Les élections de germinal an VII

Ces élections eurent lieu à la date prévue, en germinal an VII. Sur les conditions dans lesquelles se déroulèrent les assemblées, nous n'avons de renseignements que pour le IIIe arrondissement; encore ne s'agissait-il que de simples problèmes de droit, d'ailleurs complexes, posés par le remplacement à faire de deux citoyens qui avaient refusé leur élection ${ }^{81}$.

Quant aux résultats des élections, un état des membres des administrations municipales, accompagné de « renseignements sur leur patriotisme, leurs opinions et leur capacité », - celle-ci passant en dernier - dressé fin floréal-début prairial ${ }^{82}$, permet de noter que soixante-deux administrateurs, sur quatre-vingt-un seulement indiqués, plus des trois quarts, $76,54 \%$, étaient déjà en place à la veille des élections, qu'ils aient été élus l'année précédente ou qu'ils aient été nommés pour remplacer des membres démissionnaires ou destitués, mais combien parmi eux avaient été vraiment reconduits? Nous ne pouvons répondre, faute de savoir quels sont ceux que, de toute façon, le sort avait préservés du renouvellement; dix-huit seulement, en tout cas, soit $22,22 \%$, étaient des hommes nouveaux, dans la mesure où ils n'étaient pas simplement des "revenants". Ces résultats pouvaient paraître pour le Directoire un succès d'autant plus marqué que ceux qu'il avait destitués ne figuraient pas sur cette liste; c'était notamment le cas pour le 1er arrondissement. Dans trois autres, les IIe, IVe et VIIe, la municipalité avait été intégralement reconduite; dans le VIe et le IXe, elle l'avait été à raison de six officiers sur sept, de cinq encore dans le Ve et le XIIe, ainsi que dans le XIe, dont quatre qui avaient été "nommés peu de tems avant les élections» - le 5 ventôse $^{83}$-, à raison de quatre encore dans deux autres arrondissements, le Ier et le VIIIe; deux administrateurs seulement avaient été réélus dans le Xe; quant à la municipalité du IIIe, elle restait incomplète, car les questions qui s'étaient posées à son sujet n'étaient toujours pas résolues.

Pourtant une épuration avait été prévue, d'avance cette fois, à l'échelle de toute la France d'ailleurs. Dès le 19 ventôse ${ }^{84}$, le ministre de l'Intérieur, François (de Neufchâteau), avait invité les commissaires du Directoire près les administrations des départements à se "procurer les renseignemens les plus exacts sur le patriotisme, les opinions et la capacité des membres des administrations municipales et à requérir la 
suspension de ceux qui seroient reconnus indignes de la confiance dont ils (avaient) été honorés"; il fallait ainsi apprendre "à tous que le Gouvernement exerç(ait) une surveillance active et que son vœu inébranlable (était) que les fonctions publiques ne (fussent) exercées que par des républicains prononcés, également ennemis de toutes les factions, d'une moralité éprouvée et dont la capacité (pouvait) d'ailleurs assurer l'exécution des loix ", précisait-il le 21 germinal ${ }^{85}$, - on ne saurait mieux dire que les choix des citoyens étaient subordonnés aux vues du pouvoir. Le 21 floréal, le ministre prescrivit expressément aux commissaires du Directoire près le département de la Seine d'établir un «Tableau Général de la composition des administrations Municipales du Département $»^{86}$ et c'est ce tableau que, le 17 prairial, ce dernier lui adressa sous le titre qui reprenait les termes mêmes de la circulaire ministérielle ${ }^{87}$.

Le coup d'État du 30 prairial an VII

C'est alors que survint le coup d'État du 30 prairial an VII, qui vit l'éviction du Directoire de Merlin (de Thionville) et de La Revellière après l'annulation, le 28, de l'élection de Treilhard. L'épuration qui suivit fut peut-être plus ample que celle que François (de Neufchâteau) avait prévue et sans doute dans un sens quelque peu différent. Opportunément d'ailleurs, une "pétition de plusieurs citoyens du faubourg Marceau ${ }^{88}$, après avoir rendu "grâces aux Conseils" pour avoir, par leur énergie, "fait pâlir le crime", leur demandait "d'inviter le Directoire de purger les administrations municipales qui en partie (étaient) composées d'hommes indignes du nom de républicains, parce que beaucoup d'entre eux (étaient) des chefs de la révolte de vendémiaire ». De son côté, le 24 messidor, l'administration du département faisait savoir au ministre de l'Intérieur que "Les changemens salutaires opérés par les événemens du 30 Prairial dernier ne produiroient pas tout l'effet que le peuple français a(vait) droit d'attendre s'ils ne s'étendaient pas sur tous les fonctionnaires publics dont la complicité ou la faiblesse (avait) secondé la Tyrannie triumvirale ${ }^{89}$. Puis, un rapport, daté seulement de messidor sans autre précision, du nouveau ministre de l'intérieur, Quinette, adressé au Directoire, signalait que, d'après l'administration centrale du département de la Seine, dix des administrations municipales du canton de Paris étaient «frappées de la réprobation de tous les républicains", soulignant que celle du Ve arrondissement était « composée de Républicains et que celle du XIe a(vait) déjà été réorganisée » - de façon particulièrement rapide; "je propose, concluait le ministre, la destitution des membres que l'administration centrale a désignés, et leur remplacement par les citoyens qu'elle présente à cet effet »; «j'observe, notait-il, que ceux indiqués pour compléter la 10e administration municipale de Paris, sont, hormis deux seulement ${ }^{90}$, les mêmes que ceux en exercice l'an dernier et qui avoient été destitués "; il s'agissait donc d'une revanche à prendre.

L'épuration, perpétrée le 28 messidor fut cette fois massive ${ }^{91}$. Ce ne fut pas moins de soixante administrateurs, soit $71,42 \%$, qui furent destitués; trois autres ayant donné leur démission, ce sont donc soixante-trois administrateurs, très exactement les deux tiers, qui devaient être remplacés. Seule fut épargnée la 5e municipalité dont tous les membres étaient conservés; dans quatre arrondissements, les IIe, VIe, VIIe et VIIIe, tous furent indistinctement frappés ${ }^{92}$; six sur sept furent destitués dans les IVe et XIIe arrondissements, cinq dans les Xe et XIe, quatre encore dans le IXe, où deux élus avaient démissionné, ainsi que dans le Ier qui comptait également un démissionnaire, deux seulement dans le IIIe. On peut noter que trente-quatre des soixante destitués avaient été déjà élus en l'an VI, ce qui soulignait la confiance de leurs concitoyens. Au total, il s'agissait d'un important renouvellement du corps municipal; sur les quatre- 
vingt-quatre administrateurs, quarante-sept, plus de la moitié, 55,95\%, étaient des hommes nouveaux.

Les choses cependant n'étaient pas entièrement stabilisées encore. Seize citoyens en effet refusèrent leur nomination, soit le quart $-25,39 \%$. Leur remplacement ne se fit pas du jour au lendemain, d'autant plus que, cette fois encore, des citoyens pressentis refusèrent à leur tour. C'est ainsi que six administrateurs furent nommés le 29 fructidor ${ }^{93}$, cinq le 28 vendémiaire an VIII $^{94}$, quatre le 6 brumaire $^{95}$, trois enfin le 11 brumaire $^{96}$, soit dix-huit en tout. D'autres nominations résultèrent d'ailleurs de nouvelles destitutions; dans le VIe arrondissement, par exemple, trois administrateurs nommés le 28 messidor furent destitués le 28 vendémiaire pour la raison qu'ils n'offraient «pas au Gouvernement une garantie suffisante pour l'exécution des loix et du maintien de la Constitution Républicaine de l'an IIIe $»^{97}$, - cette constitution que certains se préparaient à renverser... le Directoire n'aurait-il pas toujours effectué ses choix au lendemain du 30prairial avec assez de discernement ?

De toute façon, le jeu des destitutions allait bientôt cesser; avec le coup d'État du 18 Brumaire, toutes les municipalités furent suspendues ${ }^{98}$ et bientôt de nouveau épurées ${ }^{99}$, en attendant que fût défini un nouveau régime municipal.

Comment les citoyens avaient-ils vécu toutes ces vicissitudes de l'époque directoriale ? Une seule fois, les élections avaient été à peu près respectées, celles de l'an IV; trois fois sur quatre, une épuration avait été perpétrée, tendant à devenir un système de gouvernement, en fructidor an V, en messidor-thermidor an VI et en messidor an VII, la première décidée a posteriori, la seconde sur le moment même, la troisième à l'avance, limitée en l'an VI, massive en fructidor an V et en l'an VII ${ }^{100}$. Cela ne pouvait manquer de provoquer un climat d'incertitude et de discréditer le régime. Sans doute la vie politique avait-elle été vive, à certains moments au moins, à la veille des élections, notamment celles de l'an VI, mais combien de citoyens concernait-elle? Une minorité, une quinzaine de milliers au plus et encore semble-t-il bien qu'elle se soit réduite d'une année à l'autre, même s'il est impossible de mesurer son évolution, faute de sources suffisantes. Quel a pu être le degré de détachement à l'égard d'un régime qui recourait périodiquement à l'arbitraire, qui, pour reprendre les termes utilisés par Antonelle dès l'an VI, "nomme et révoque, place et destitue, replace et remplace ${ }^{101}$, qui, selon Aulard, en contrariant l'application du principe électif, rendait «illusoire le droit accordé aux citoyens de nommer les fonctionnaires » ?102. Peut-on, avec Aulard encore, considérer que, "quand la constitution de l'an VIII supprima cette élection des fonctionnaires, on ne fut ni surpris ni affligé de perdre un droit dont on n'usait presque plus $»^{103}$ ? On peut noter que, pour le plébiscite de l'an VIII, le nombre des votants, d'après les relevés de $\mathrm{C}$. Langlois ${ }^{104}$ fut, à Paris, de 28400 , près du double des votants pour l'élection de thermidor an IV, mais sur combien d'ayants droit, alors que, si tous les citoyens étaient appelés à voter, il n'était pas précisé s'il fallait définir la qualité de citoyen selon la Constitution de l'an III ou dans le sens le plus large? L'empressement des citoyens restait de toute façon limité; pour une population parisienne estimée à 457756 en l'anIX ${ }^{105}$, la participation était de 5,18\%, après avoir été, il est vrai, de 2,61\% seulement aux élections de l'an IV, pour une population estimée en vendémiaire suivant à $556304^{106}$. Sans doute, si beaucoup de citoyens, déçus par le régime directorial, étaient prêts à accueillir sans regret son renversement, la plupart restaientils dans l'expectative face à celui qui allait le remplacer. 


\section{ANNEXES}

Annexe I - Les douze arrondissements de Paris et leurs divisions (anciennes sections)

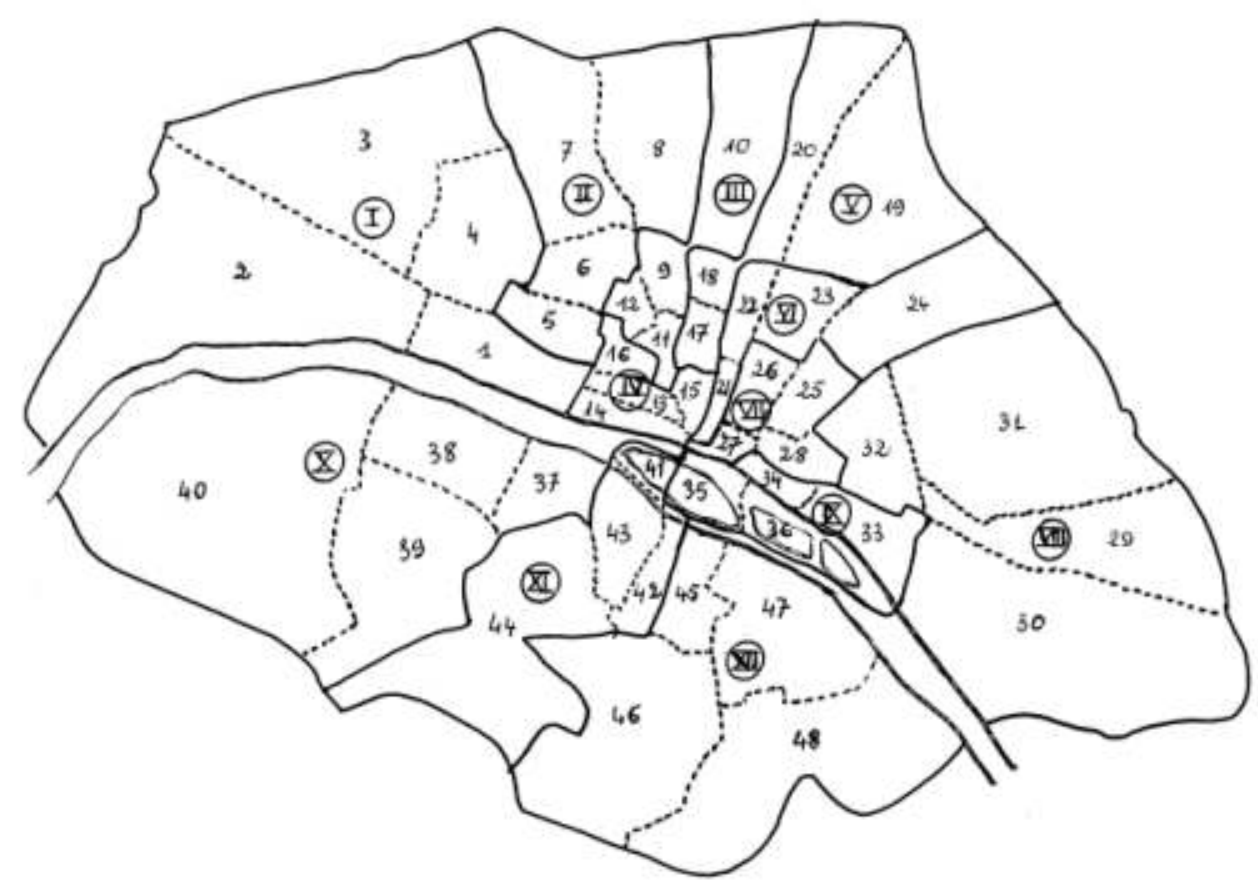

Les arrondissements de Paris (1795)

Distribution des sections entre les 12 arrondissements

\begin{tabular}{|l|l|l|l|l|}
\hline Ier ar & 1) Tuileries & VIIe ar & 25) L’Homme-Armé (Enfants-Rouges) \\
\hline \hline & 2) Champs-Élysées & & 26) Réunion (Rue-Beaubourg) \\
\hline \hline & 3) Roule & & 27) Arcis \\
\hline \hline & $<\mathrm{p}$ & & \\
\hline
\end{tabular}

\section{NOTES}

1.Titre VII, articles 183 à 185 (le Moniteur, t. 25, n 340, pp. 565-576). Les arrondissements de Paris avaient été constitués par la loi du 7 fructidor an II concernant les comités révolutionnaires (Bulletin des lois, t. I, An II, $n^{\circ} 247$ ). Voir la carte des 12 arrondissements, annexe $n^{\circ} \mathrm{I}$.

2.Bulletin des lois, t. 6, An IV, vendémiaire-brumaire, $\mathrm{n}^{\circ} 1160$.

3.Ibid., $\mathrm{n}^{\circ} 1205$.

4.Ibid., An IV, brumaire, ventôse, $\mathrm{n}^{\circ} 133$.

5.Archives nationales (par la suite A.N.), AF III, 302. 
6.Par un message reçu le 15 nivôse par le Conseil des Cinq-Cents, d'après l'Ami des lois, $\mathrm{n}^{\circ}$ 153, du 20 nivôse an IV.

7.D'après un autre tableau, postérieur au 5 floréal, puisqu'il y est à fait mention de démissions reçues ce même jour ; « Organisation des Autorités Constituées.

Département de la Seine. Pluviôse an IV » (A.N., F1b II/Seine/ 23).

8.D'après une pièce intitulée : «Époque du 26 pluviôse l'an 4 . Relevé des nominations qui restent à faire pour compléter les 12 Municipalités de Paris » (ibid., AF III, 302).

9.Deux dans le XIe arrondissement, trois dans le XIIe (ibid., F1b II/Seine/22).

10.Deux démissions furent données le 2 messidor dans le Ier arrondissement (ibid., $\mathrm{F} 1 \mathrm{~b}$ II/ Seine/ 23), deux acceptées le 18 dans le Ve (ibid., F 1 b II/Seine/22).

11.Voir les procès-verbaux des assemblées primaires pour le premier scrutin (ibid., B $1 / 16)$.

12.D'après un « Tableau de distribution des Assemblées primaires des 12 arrondissements du canton de Paris ", du 19 messidor an IV (A.N., F20, 381).

13.« Extrait du registre du Conseil de Ville. Séance du 2 Aoust 1790, prorogée dans la nuit du 3 » (Archives de la Seine, par la suite A.S., VD 4 ). Il y avait alors 77590 ayants droit de vote en octobre 1790, d'après un «État certifié par le Directeur des Impositions de la Ville et fauxbourgs de Paris, le 2 octobre 1790 » joint à une « Lettre du Procureur de la Commune aux Citoyens actifs des Sections de la Ville de Paris (...) »(A.N. 69). 14.D'après le procès-verbal du Corps Municipal (S. Lacroix, Actes de la Commune de Paris pendant la Révolution, Paris, 1884-1914, deux séries, 1re série, t. 8, p. 70).

15.« Extrait du registre du Conseil de Ville du 29 septembre 1790 », (Bibliothèque historique de la Ville de Paris, 104.097, impr. In-4² 2p.) et « Extrait (...) du 2 octobre $1790 »$ (ibid., Mss.803, p pe $^{\text {ce } 65) . ~}$

16. La $7^{\mathrm{e}}$ assemblée du IVe arrondissement et la $10^{\mathrm{e}}$ assemblée du VIe arrondissement 17.La 9e assemblée du IIIe arrondissement, la 7e assemblée du VIe et la 18e assemblée du XIIe.

18.Dans Le Nombre et la Raison. La Révolution française et les élections, Paris, 1993, pp. 339 et sq. Pour le comportement des électeurs parisiens en 1790-1791, voir M. Genty ; « Les élections parisiennes pendant la période constitutionnelle de la Révolution française ", dans Voter et élire à l'époque contemporaine, Cahiers de l'I.H.C., n 4, 1999, Éditions universitaires de Dijon, Direction A. Bleton-Ruget et S. Wolikow. Pour l'ensemble des arrondissements en thermidor an IV, voir le tableau annexe $n^{\circ} 2$.

19.La 9e assemblée du IIIe arrondissement et la 6e assemblée du Xe, avec 33,07\% et $33,15 \%$ respectivement.

20.Dans la 8e assemblée du IIIe arrondissement, les 3e et 10e assemblées du XIIe arrondissement respectivement.

21.Dans la 4e assemblée du IVe arrondissement et la 7e assemblée du Xe respectivement.

22.Dont cinq dans les IVe et Ve arrondissements, deux dans le VIe et un dans le XIe.

23.Conformément à l'article 9 du titre III de la loi du 25 fructidor an II, citée par la 1re assemblée du Ier arrondissement.

24.A.N., F 1 b II/ Seine/23.

25.Dans la 8e assemblée du IIIe arrondissement et dans la 10e assemblée du Xe.

26.Dans la 8e assemblée du XIe arrondissement et dans la 3e assemblée du IIe.

27.Dans deux assemblées du Xe arrondissement; dans la 3e, un citoyen avait demandé

réparation pour une insulte dont il avait été l'objet et, dans la 10e, les bulletins avaient dû être brûlés à la suite d'une erreur quant à sa circonscription. 
28.A.N., F 1 b II/ Seine/23.

29.Dans la 3e assemblée du XIIe arrondissement. Dès le 4 thermidor, le Journal de Paris avait noté, à propos de deux assemblées visitées, que, si les assemblées avaient été " très peu nombreuses ", « Heureusement les votants sont tous d'honnêtes gens ", ajoutant : « On assure qu'ils ont renommé une grande partie des magistrats institués par le gouvernement depuis l'épuration » (t. 37, n 304, p. 1215).

30.Par la loi du 25 fructidor an IV (Bulletin des lois, t. 6, an IV, vendémiaire-brumaire, $\mathrm{n}^{\circ}$ 1162).

31.A.S., VD ${ }^{\mathrm{X}}, \mathrm{n}^{\text {os }} 5405$ à 5412.

32.Texte cité $n .12$.

33.En l'an IV, il n'y avait plus que deux officiers municipaux à élire sur sept, ce qui pouvait faire paraitre le second scrutin de faible importance.

34.D'après le Journal de Paris, du 13 germinal, jamais les votants n'avaient été nombreux; "On en avait compté près de 30 mille ", affirmait-il, mais sans préciser sur quelles bases il donnait ce chiffre global qui, au demeurant, concernait les élections préalables à celles des administrateurs municipaux. Par ailleurs, certains journaux, la Chronique de Paris ressuscitée, dans son $\mathrm{n}^{\circ} 5$ du 3 germinal, et le Journal de Paris, dans son $\mathrm{n}^{\circ} 193$ du 13 germinal, s'accordent au moins sur deux points, le caractère paisible des assemblées et le choix de " vendémiaristes " pour la composition des bureaux, sauf, d'après le premier journal, pour la présidence de l'assemblée du Luxembourg, obtenue par un ancien jacobin, Dorat-Cubières, qui avait été électeur de 1792 et membre de la Commune jusqu'en germinal an II. De son côté, le Journal des Hommes Libres, dans son $\mathrm{n}^{\circ}$ 168 du 3 germinal, p. 700, notait que, « jusqu'ici, une tranquillité un peu morne les accompagne (les assemblées primaires). Il semble qu'on se tâte ; cependant, dans quelques sections reparaissent hardiment les électeurs de vendémiaire (...) », et le journal invitait les patriotes à « ne pas hésiter plus » à se montrer.

35.L'un dans le Ve arrondissement, où une démission avait nécessité un remplacement le 2 brumaire an $\mathrm{V}$, les deux autres dans le VIIIe arrondissement, où deux noms avaient disparu, nous ne savons dans quelles conditions, remplacés par un seul autre, de sorte que la municipalité était restée incomplète lors de la publication de l'Almanach.

36.Il s'agit de la « loi contenant des mesures de salut public relativement à la conspiration royaliste " (Bulletin des lois, t. 9, An V, 1er semestre, $\mathrm{n}^{\circ}$ 1400).

37.A.N., F1b II/Seine/8.

38.Les VIIe, VIIIe, IXe et XIIe arrondissements.

39.Les IIe, IIIe et IVe arrondissements.

40.Les Ier et IVe arrondissements.

41.A.N., F 1 b II/ Seine/8.

42.Ibid., F 1 b II/Seine/24.

43.Ibid.

44.Ibid., F 1 b II/ Seine/ 18.

45.Ibid., F1b II/Seine/ 23.

46.L'un d'eux, Durouzy, dans le VIIe arrondissement, le 1er frimaire, l'autre, Tessier, dans le Xe, le 7 pluviôse - c'est lui qui n'avait été nommé que le 16 vendémiaire an $\mathrm{V}$ (ibid., F1b II/Seine/24).

47.L'un, Haupois, dans le VIe arrondissement, le 5 frimaire; l'autre, Roche, dans le Ve arrondissement, le 15 ventôse (ibid.).

48.Le 17 nivôse, destitution motivée par le fait que l'intéressé, Leclerc, s'était , la Théophilanthropie en l'occurrence (ibid.). 
49.Bulletin des lois, t. 9 An VI, nos 1723, 1729 et 1745 respectivement.

50.D'après le ministre de l'Intérieur, en réponse à une question posée par une ou plusieurs sections.

51.A.S., VD ${ }^{\mathrm{X}}, \mathrm{n}^{\text {os }} 54 \mathrm{I} 3$ à $54 \mathrm{I} 6$ et 5449 à 5466.

52.Loi citée n. 23.

53.D’après des « Notes importantes sur la formation de la municipalité du Ier arrondissement » (A.N., F 1b II/ Seine/24).

54.Il y en eut 145 puis 135 dans la 10e assemblée contre 50, 38 et 53, 99 puis 74 dans la 12 e contre 51,51 et 38 , soit environ trois à quatre fois et environ deux fois respectivement.

55.Les 1re, 2e, $4 \mathrm{e}$ et $8 \mathrm{e}$ assemblées.

56.A.N., AF III, 260.

57. À la suite du procès de Vendôme contre les babouvistes.

58. Celle de la division du Pont-Neuf.

59.Dans le VIe arrondissement.

60.Les deuxièmes assemblées des Ier et VIe arrondissements et les 2e et 5e assemblées $\mathrm{du}$ Xe.

61.Les assemblées $n^{\circ} 1$ du IIe arrondissement, $n^{\circ} 2$ du IIIe, $n^{\circ} 1$ du Ier et $n^{\circ} 5$ du XIe.

62.La deuxième assemblée du VIe arrondissement.

63. Celles de Charonne et de la Roquette.

64. "Tableau des membres composant les administrations Municipales du Département de la Seine en Messidor an VI, avec Notes et Renseignements », en vue de leur épuration (A.N., F 1b II/ Seine/ 25).

65.Texte cité n. 53.

66.Dans le texte cité n. 64 .

67.Lors du dépouillement du second scrutin, il s'était trouvé un bulletin qui contenait sept noms alors qu'il n'en fallait plus que cinq et « l'assemblée, au lieu de faire effacer les deux derniers »avait effacé « les noms des deux citoyens déjà nommés » et aucune mention n'en avait été portée au procès-verbal.

68. La première et la cinquième.

69. Texte cité n. 64 .

70.Il y en avait eu un seul dans le VIIe arrondissement, deux dans le IIe, trois dans le IIIe, le VIIIe et le Xe.

71.Voir plus loin, note 79.

72.Deux dans le IIIe arrondissement, trois dans le IVe.

73.L'un d'eux dans le IIIe arrondissement, un autre dans le Ve, parce qu'il répugnait à appliquer les mesures de surveillance ordonnées par le Département contre les prêtres réfractaires; un autre démissionna dans le Ve arrondissement, deux dans le IXe, un dans le Xe et quatre dans le seul VIIe arrondissement, dont trois avaient fomenté des troubles lors des élections de germinal an VI.

74.D’après la lettre ci-dessus du commissaire du Directoire près le Département au ministre de l'Intérieur, jointe au tableau cité n. 64 .

75.Ibid.

76.Les IVe, VIe, VIIe, VIIIe, IXe et XIIe arrondissements.

77.Certaines étaient sans doute plus ou moins fondées; plusieurs d'entre eux en tout cas avaient exercé des fonctions officielles dans la période consécutive au 10 août 1792 et sous la Terreur; deux en particulier, Perdry et Bunon, avaient été électeurs de 1792. Sur l'engagement révolutionnaire de cinq d'entre eux, Perdry, Bunon, Marquet, Brullart et 
Moussard, voir A. Soboul et R. Monnier, Répertoire du personnel sectionnaire parisien de l'an II, Paris, 1985, pp. 72, 58, 78, 48 et 86 respectivement. Quant à Foloppe, il avait été arrêté en brumaire an II et libéré en fructidor an IV (A.N., F7/4708). Pour l'orthographe de Perdry, nous avons adopté celle que donne sa propre signature.

78.Comme le notait, le 27 thermidor, un rapport du ministre de l'Intérieur au Directoire (A.N., F1b II/Seine/23).

79.Le 24 messidor pour l'un d'eux, Plongenet, le 14 thermidor pour les trois autres. 80.L'arrêté, pris par le Département le 14 thermidor an VI, n'avait toujours pas été mis à exécution à la fin de nivôse an VII, ces citoyens « ayant depuis ce tems suivi une marche plus mesurée » (A.N., F 1b II/Seine/23).

81.Ibid., F 1 b II/Seine/25.

82. "État nominatif des Membres des administrations Municipales avec des renseignements sur leur patriotisme, leur moralité, leurs opinions et leur capacité », envoyé le 17 prairial par le commissaire du Directoire près l'administration centrale du département de la Seine au ministre de l'Intérieur (ibid.).

83.D'après une lettre du commissaire du Directoire au ministre de l'Intérieur, du 2 floréal an VI (ibid.).

84.Ibid., F1 A/23.

85.Ibid.

86.D'après la lettre du commissaire du Directoire jointe à l'état demandé (ibid., F 1b II/ Seine/25).

87.Cf. n. 82.

88.Pétition transmise le 23 messidor au nouveau ministre de l'Intérieur, Quinette (ibid.).

89.Ibid.

90.Ils s'appelaient Naigeon et Rolin.

91.A.N., 1b II/Seine/25, an VII.

92.Dans le IIe et le VIIe arrondissements, en particulier, tous les administrateurs en place au moment des élections avaient été reconduits par leurs concitoyens. À noter qu'étaient également maintenus, dans le Ier arrondissement, Moussard et Brullart, qui l'avaient été déjà en messidor an VI.

93.Trois dans le IXe arrondissement et trois dans le XIIe.

94.Trois dans le VIe arrondissement, un dans le IXe et dans le XIIe encore.

95.Deux dans le IIIe et dans le IVe.

96.Dans le VIIe arrondissement.

97.A.N., F 1 b II/Seine/25.

98.Cf. la « Loi du 16 frimaire relative aux actes reçus par le commissaire du Gouvernement, pendant la suspension des douze municipalités de Paris, les 18, 19, 20 et 21 brumaire an VIII » (Bulletin des lois, An VIII, 1er vendémiaire-27 nivôse, $n^{\circ} 3450$ ).

99.Cette épuration fut, elle aussi, massive; 43 officiers municipaux, plus de la moitié $51,1 \%$ - furent destitués, dont six dans le XIIe arrondissement, cinq dans quatre autres, les Ier, Ve, VIIIe et XIe, quatre dans le VIe et dans le VIIe, trois dans le IIe et dans le IVe, un dans le IIIe, aucun dans le IXe (A.N., F1b II/Seine/25, an VIII).

100.Il y avait eu 57 destitutions en fructidor an V, 12 plus une provisoire en messidorthermidor anVI et 60 après le 30 prairial an VII.

101.Cité par P. Serna, Antonelle, aristocrate révolutionnaire.1747-1817, Paris , 1997, p. 375.

102. Histoire politique de la Révolution. Origine et développement de la démocratie et de la république. 1789-1804, Paris, 1901, p. 595. 
103.Id.

104. "Le plébiscite de l'an VIII ou le coup d'État du 18 pluviôse an VIII », Annales historiques de la Révolution française, 1972, nos 207 à 209, pp. 237-238.

105.« Département de la Seine. Dénombrement de la population des communes du département de la Seine, terminé au commencement de l'an IX »(A.N., F 20, 381). 106.D'après un dénombrement effectué par les comités de bienfaisance des sections parisiennes à la demande du ministre de l'Intérieur, formulée le 14 vendémiaire an IV (A.N., F 20, 123-124); nous avons repris les chiffres établis par M. Reinhard dans la Nouvelle histoire de Paris. La Révolution, 1789-1799, Paris, 1971, p. 434.

\section{RÉSUMÉS}

Les élections municipales à Paris sous le Directoire, jusqu'ici négligées, en raison peut-être de la rareté des sources, ne sont pas cependant sans intérêt. Sans doute n'ont-elles attiré qu'une faible minorité de citoyens, proportionnellement plus faible même qu'au début de la Révolution, bien qu'elles aient été parfois âprement disputées, notamment en l'an VI. Cette minorité semble même s'être amenuisée d'une année à l'autre, vraisemblablement du fait des épurations, le plus souvent massives, qui suivirent chacune de ces élections, sauf les premières, celles de l'an IV ; devenues systématiques, n'ont-elles pas préparé les esprits à l'abandon du principe électoral?

\section{The Municipal Elections in Paris during the Directory.}

The municipal elections in Paris during the Directory, neglected until now perhaps on account of the paucity of sources, are not without interest. To be sure, they attracted only a small minority of citizens, proportionately even fewer than at the beginning of the Revolution, although they were sometimes bitterly contested, especially in Year Vl. This minority seems to have dwindled even further from one year to the next, probably as a result of the often massive purges which followed each of these polls, apart from the first, the poll of Year IV. Once they became systematic, people may well have lost interest in the principle of voting altogether.

\section{Le elezioni municiali a Parigi soto il Direttorio}

Le elezioni municipali a Parigi sotto il Direttorio, finora trascurate, forse per via della scarsità delle fonti, non sono tuttavia prive di interesse. Indubbiamente non hanno attratto se non una ridotta minoranza dei cittadini, propozionalmente più ristretta che all'inizio della Rivoluzione pur essendo talvolta aspramente combattute, specie nell'anno VI. Questa minoranza anzi pare essersi ridotta da un anno all'altro, verosimilmente a causa delle epurazioni, il più delle volte massiccie consecutive ad ognuna di queste elezioni, tranne le prime, quelle dell'anno IV; diventate sistematiche non avranno preparato gli animi all'abandono del principio elettorale?

Quizas por falta de fuentes, fueron poco estudiadas las elecciones municipales en Paris durante el Directorio. Aunque muy reñidas, como en el año VI, atrayerón una poqueña minoría de ciudadanos, menos que en los principios de la Revolución. Esta minoría se reducc de una año para otro por las depuraciones que siguieron cada una de las elecciones, excepto las primeras, en el año IV, preparando así la gente al abandono del principio electoral.

Die Kommunalwahlen in Paris zur Zeit dies Direktoriums.

Die Pariser Kommunalwahlen zur Zeit des Direktoriums, die bisher vielleicht wegen der seltenen 
Quellen vernachlässigt worden sind, sind immerhin betrachtenswert. Zwar haben sie eine sehr geringe Zahl von Bürgern - sogar eine kleinere als am Anfang der Revolution - mobilisiert, obwohl sie manchmal, wie im Jahre VI, Gegenstand heftiger Diskussionen gowesen sind. Wahrscheinlich wegen der oft massiven Säuberungen, die jeder dieser wahlen folgten, scheint diese Minderheit sogar von Jahr zu Jahr immer kleiner geworden zu sein. Haben diese systematisch gewordenen Säubrungen die Menschen schließlich nicht dazu veranlaßt, auf das Wahlprinzip zu verzichten? 